\title{
Is the Double Contour Sign Specific for Gout? Or Only for Crystal Arthritis?
}

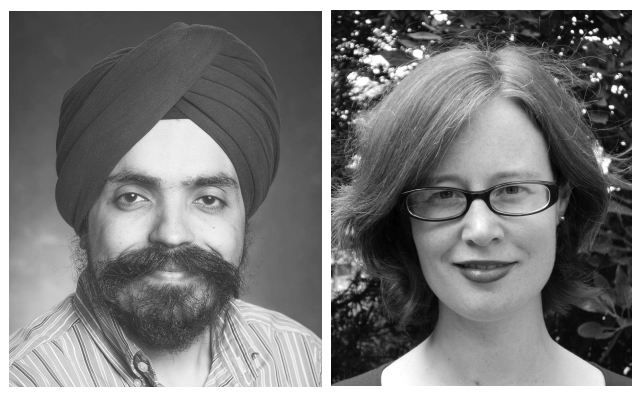

In this issue of The Journal, Löffler, et al describes a study assessing the sonographic double contour (DC) sign in gout, calcium pyrophosphate crystal deposition (CPPD), and other arthritides ${ }^{1}$. They investigated the diagnostic value of the DC sign alone and in combination with Doppler signals and serum uric acid (SUA) levels in patients presenting with acute arthritis by examining 225 acutely inflamed joints. Cartilage enhancements presenting as a parallel line to the bony articular surface were defined as the DC sign. All patients underwent synovial fluid analysis that was used to make the diagnosis of gout or CPPD or other arthritides, independent of the joint ultrasound (US) findings. The sensitivity of the DC sign for crystal arthritis was $85 \%$ and specificity was $80 \%$. Its specificity for gout was $64 \%$, and for CPPD 52\%. The combination of DC sign with hypervascularization in Doppler studies and elevated SUA levels increased specificity for gout to $90 \%$ and resulted in a 7 -fold increase of likelihood of gout $(\mathrm{p}<0.01)$, albeit at the expense of sensitivity (42\%). The study has several strengths, including use of a large patient sample, confirmation of diagnosis by crystal examination, and appropriate acute arthritis controls. The study findings must be interpreted considering potential limitations and these findings need to be compared and contrasted with those from other studies of US in gout.

A striking finding of this study reporting the use of US in real-world clinical practice was the inability of the DC sign to differentiate between gout and CPPD. In previous US publications, the differential cartilage appearances of monosodium urate (MSU) and CPPD have been elegantly described, with MSU crystals overlying the articular cartilage leading to the DC sign in gout, and CPP crystals deposited within articular cartilage with a different appearance ${ }^{2,3,4}$. Of note, the study by Löffler and colleagues considered only the presence of DC sign and Doppler flow, but not tophi or fibrocartilage pathology, which may explain the low accuracy of US findings. These factors do not, however, explain the low specificity of DC sign for gout compared to CPPD. Six trained ultrasonographers, who each had $\geq 2$ years of training but various degrees of expertise, performed the examinations. Use of 6 examiners leads to interobserver variability that might have contributed to increased inaccuracy. The authors used the presence of phagocytized urate crystals as the diagnostic standard for gout. They do not describe whether the presence of extracellular urate crystals only was also considered diagnostic for gout.

The authors suggest that one reason their results differ from those of other reports is that previous studies have had very few control subjects with CPPD and were limited in many cases to the examination of the first metatarsophalangeal (MTP) or the knee joint, as compared to examination of only the acutely inflamed joint in their case. The majority of the joints examined in this study were knees, with fewer than 10 first MTP joints. It is currently unclear whether a diagnostic approach that involves scanning only a single affected joint provides sufficient accuracy for gout diagnosis. Recent work by Naredo and colleagues ${ }^{5}$ indicates that systematic bilateral scanning for hyperechoic aggregates at the radiocarpal joint, patellar tendon and triceps tendon, and DC sign in 3 articular cartilages (i.e., first metatarsal, talar, and second metacarpal/femoral) is required for adequate diagnostic accuracy; this approach had a sensitivity of $85 \%$ and specificity of $83 \%$. In contrast, the sensitivity and specificity for DC sign at the femoral condyle was $42 \%$ and $100 \%$, respectively, and at the first metatarsal dorsal cartilage was $62 \%$ and $98 \%$, respectively ${ }^{5}$. Thus, the variation in diagnostic accuracy between different studies may reflect not just expertise in musculoskeletal US, but also the characteristics of the control population, and the sites and number of joints scanned.

Other studies have described false-positive DC sign due to normal hyperechoic aspect of the synovium, the presence of joint effusion (which induces a posterior-wall echo reinforcement), or thin cartilage ${ }^{6}$. To avoid artifact, some US experts have recommended that the DC sign should be defined as a "hyperechoic irregular enhancement of the chondrosynovial margin of the cartilage that is independent

See Differentiating gouty arthritis from CPPD and other arthritides, page 513

Personal non-commercial use only. The Journal of Rheumatology Copyright (c) 2015. All rights reserved. 
of the insonation angle of the US beam"5. A false-negative DC sign may be due to poorly visualized joints with thin cartilage (tarsal joints) or damaged cartilage, as in osteoarthritis (OA). The DC sign has also been described in up to $29 \%$ of individuals with asymptomatic hyperuricemia ${ }^{7,8}$. It seems likely that this finding in asymptomatic hyperuricemia represents true subclinical MSU crystal deposition, because the DC sign was observed at much lower frequency in normouricemic controls $(0-5 \%)$ in the same studies ${ }^{7,8}$. Further, another study of 26 asymptomatic individuals with hyperuricemia, of whom 9 had urate crystals documented from joint fluid, DC, or hyperechoic cloudy area, had a positive predictive value of $82 \%$, sensitivity of $100 \%$, and specificity of $88 \% 9$.

What then are the implications of the DC sign controversy? As often happens, when a new sign or test is developed/discovered for a condition, it seems more specific than it actually is. The true estimation of the specificity and positive predictive value of a new test or sign is determined when appropriate controls have been recruited into subsequent studies. Also, the accuracy statistics of the DC sign would vary by the clinical severity of the gout cases (early presentation with low MSU crystal burden vs longstanding, untreated or tophaceous disease with high MSU crystal burden), type of joint/s examined, number of joints examined, and the presence of joint inflammation at the time of examination (acute symptomatic vs chronic phase). It is worth noting that the authors did not report the results of plain radiographs; the presence of cartilage calcification at characteristic sites (triangular fibrocartilage, menisci of the knee) is characteristic of CPPD and greatly assists in the diagnosis of this condition. It seems likely that inclusion of plain radiographic cartilage calcification within the prediction models would lead to a more accurate diagnostic rule.

This study improves our understanding of the emerging role of US for the diagnosis of gout, in particular, its differentiation from conditions that mimic gout, such as CPPD, OA, rheumatoid arthritis, and psoriatic arthritis. As our understanding of the role of the DC sign evolves, so will its importance and contribution to the diagnosis of gout. This study highlights the potential difficulty of translating US research findings into routine clinical practice, and emphasizes the need for standardization of US methodology, to ensure that it is a reliable diagnostic tool for gout. Such standardization exercises will require relevant arthritis controls, including those with other forms of crystal arthritis. In clinical practice, the issue of diagnosis is most challenging soon after first presentation of arthritis, and it is in this situation, rather than in patients with well-established disease with numerous other clinical features of gout, that careful scrutiny is needed to understand the true accuracy of the DC sign for gout diagnosis. In the meantime, the findings of this study reinforce the conclusion that if there is clinical uncertainty, synovial fluid examination is still required for diagnosis of both gout and CPPD.
JASVINDER A. SINGH, MBвs, MPH,

Medicine Service,

Birmingham VA Medical Center,

Department of Medicine at School of Medicine, and

Division of Epidemiology at School of Public Health, University of Alabama,

Birmingham, Alabama; and

Department of Orthopedic Surgery,

Mayo Clinic College of Medicine,

Rochester, Minnesota, USA;

NICOLA DALBETH, MD, FRACP,

University of Auckland, and

Auckland District Health Board,

Auckland, New Zealand.

Supported by research funds from the Division of Rheumatology at the University of Alabama at Birmingham and the resources and use of facilities at the Birmingham VA Medical Center. Dr. Singh is supported by grants from the Agency for Health Quality and Research Center for Education and Research on Therapeutics (AHRQ CERTs) U19 HS021110; National Institute of Arthritis, Musculoskeletal and Skin Diseases (NIAMS) P50 AR060772 and U34 AR062891; National Institute of Aging (NIA) U01 AG018947; and National Cancer Institute (NCI) U10 CA149950; and research contract CE-1304-6631 from Patient Centered Outcomes Research Institute (PCORI). Dr. Dalbeth is supported by the Health Research Council of New Zealand (09/111D).

Address correspondence to Dr. J.A. Singh, University of Alabama, Faculty Office Tower 805B, 510 20th St. S, Birmingham, Alabama 35294, USA.

\section{REFERENCES}

1. Löffler C, Sattler H, Peters L, Löffler U, Uppenkamp M, Bergner R. Distinguishing gouty arthritis from calcium pyrophosphate disease and other arthritides. J Rheumatol 2015;42:513-20.

2. Grassi W, Meenagh G, Pascual E, Filippucci E. "Crystal clear"-sonographic assessment of gout and calcium pyrophosphate deposition disease. Semin Arthritis Rheum 2006;36:197-202.

3. Thiele RG, Schlesinger N. Diagnosis of gout by ultrasound. Rheumatology 2007;46:1116-21.

4. Filippucci E, Riveros MG, Georgescu D, Salaffi F, Grassi W. Hyaline cartilage involvement in patients with gout and calcium pyrophosphate deposition disease. An ultrasound study. Osteoarthritis Cartilage 2009;17:178-81.

5. Naredo E, Uson J, Jimenez-Palop M, Martinez A, Vicente E, Brito E, et al. Ultrasound-detected musculoskeletal urate crystal deposition: which joints and what findings should be assessed for diagnosing gout? Ann Rheum Dis 2014;73:1522-8.

6. Ottaviani S, Bardin T, Richette P. Usefulness of ultrasonography for gout. Joint Bone Spine 2012;79:441-5.

7. Howard RG, Pillinger MH, Gyftopoulos S, Thiele RG, Swearingen CJ, Samuels J. Reproducibility of musculoskeletal ultrasound for determining monosodium urate deposition: concordance between readers. Arthritis Care Res 2011;63:1456-62.

8. Pineda C, Amezcua-Guerra LM, Solano C, Rodriguez-Henriquez P, Hernandez-Diaz C, Vargas A, et al. Joint and tendon subclinical involvement suggestive of gouty arthritis in asymptomatic hyperuricemia: an ultrasound controlled study. Arthritis Res Ther 2011;13:R4

9. De Miguel E, Puig JG, Castillo C, Peiteado D, Torres RJ, Martin-Mola E. Diagnosis of gout in patients with asymptomatic hyperuricaemia: a pilot ultrasound study. Ann Rheum Dis 2012;71:157-8.

J Rheumatol 2015;42:353-4; doi:10.3899/jrheum.141322 